\title{
Resident Arterial Cells and Circulating Bone Marrow-Derived Cells both Contribute to Intimal Hyperplasia in a Rat Allograft Carotid Transplantation Model
}

Yi Hea Xin Xub Ting Zhu ${ }^{b}$ Min Tang ${ }^{a}$ Ju Meia Yi Sib

aDepartment of Cardiovascular Surgery, Xinhua Hospital Affiliated to Shanghai Jiao Tong University School of Medicine, 'Department of Vascular Surgery, Zhongshan Hospital Fudan University, Shanghai, China

\section{Retraction Statement see Next Page}




\section{Retraction Statement}

He Y, Xu X, Zhu T, Tang M, Mei J, Si Y, entitled "Resident Arterial Cells and Circulating Bone Marrow-Derived Cells Both Contribute to Intimal Hyperplasia in a Rat Allograft Carotid Transplantation Model" [Cell Physiol Biochem 2017;42(4):1303-1312. DOI: 10.1159/000478959].

Due to some critical technical issue, we have obtained different results in recent studies. To avoid any negative impact on the research of others in this field, after serious consideration, we have decided to retract this paper for now. We will add more convincing and reproducible data in future.

We are sorry for this decision and sincerely appreciate the efforts of the publishers, editors and reviewers. 